\title{
Temperature-Scanning Reaction Protocol Offers Insights into Activation Parameters in the Buchwald-Hartwig Pd-Catalyzed Amination of Aryl Halides
}

\author{
Olivia P. Schmidt and Donna G. Blackmond*
}

Cite This: ACS Catal. 2020, 10, 8926-8932

Read Online

ACCESS | Lلll Metrics \& More | 国 Article Recommendations | sl Supporting Information

ABSTRACT: A temperature-scanning reaction (TSR) protocol allows deconvolution of the driving forces of concentration and temperature in a single experiment, demonstrated here for the Buchwald-Hartwig amination reaction using different amine substrates that exhibit different ratedetermining steps. An Eyring analysis reveals that the observed reactivity differences between 1-hexylamine and benzophenone hydrazone are related primarily to the different contributions of activation entropy in the two cases. This TSR protocol combined with other in situ kinetic methodologies including reaction progress kinetic analysis and variable time normalization analysis provides a rapid and comprehensive mechanistic picture of complex multistep catalytic reactions.

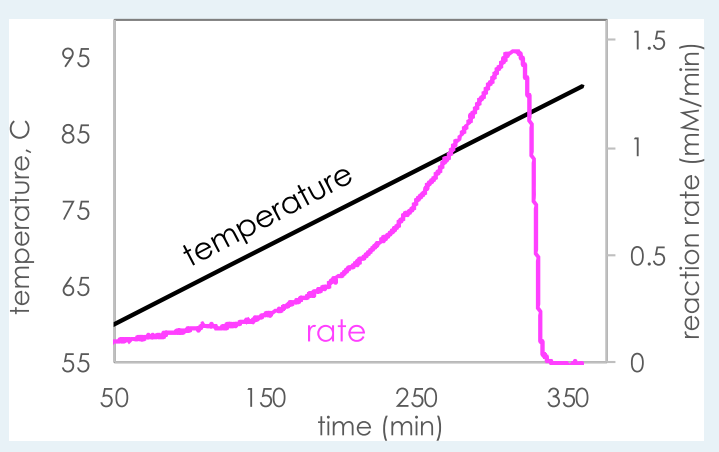

KEYWORDS: Pd catalysis, reaction progress kinetics, Eyring equation, Arrhenius equation, temperature-scanning reactions,

Buchwald-Hartwig amination

\section{INTRODUCTION}

Experimental mechanistic analysis of complex catalytic reactions is aided by global kinetic experiments that can help to propose a plausible catalytic cycle, including the reaction's rate-determining step and the catalytic resting state. Such proposals may then be tested by using spectroscopic, structural, and computational investigations. Recent developments in the use of in situ tools for reaction monitoring expedites mechanistic understanding by extracting more information from fewer experiments. For example, the methodology of reaction progress kinetic analysis (RPKA), ${ }^{1}$ in which concentration variables are continuously changing over the course of the reaction, provides information in a single experiment that would require multiple separate reactions using classical kinetic analysis.

A number of recent reports ${ }^{2-4}$ have highlighted how the reaction temperature may be treated as a continuously changing parameter over the course of a reaction, even though reactant concentrations are also changing. The ability to analyze the temperature dependence of a complex multistep catalytic reaction in the same experiment in which concentration dependencies are probed in these data-dense experiments has the potential to accelerate both the fundamental understanding and practical applications. In the present study, we demonstrate this temperature-scanning reaction (TSR) protocol for two examples of Pd-catalyzed Buchwald-Hartwig amination of aryl halides ${ }^{5}$ with 1-hexylamine $\mathbf{2 a}$ and benzophenone hydrazone $\mathbf{2} \mathbf{b}$ (Scheme 1 ).
Scheme 1. Buchwald-Hartwig Amination Catalyzed by $\operatorname{Pd}($ binap)

$$
\text { (a) or } \mathbf{4 b}
$$

\section{BACKGROUND}

The reactions of Scheme 1 have been studied comprehensively by our group, in both separate and competitive reactions, but typically under isothermal conditions. ${ }^{6}$ As shown in Figure 1, and as has been shown previously, ${ }^{6}$ the reaction of 2 a proceeds faster than that of $\mathbf{2} \mathbf{b}$ at the same catalyst concentration and lower temperatures, with the former case obeying overall firstorder dependence on $[\mathrm{ArX}]$, while the latter obeys overall

Received: April 29, 2020

Revised: July 10, 2020

Published: July 14, 2020 


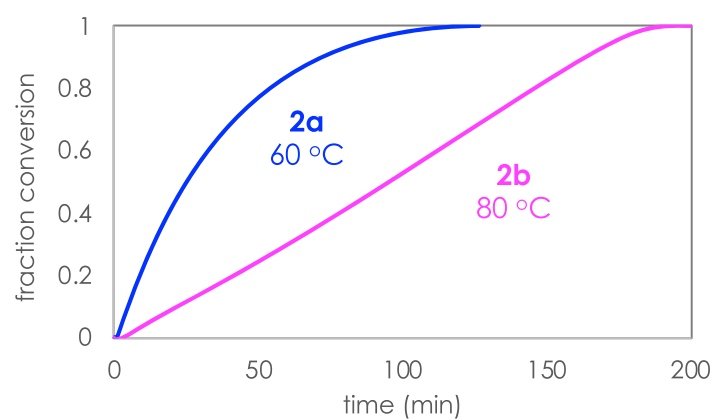

Figure 1. Fraction conversion for the reaction of Scheme 1 under isothermal conditions with $[\mathbf{1}]_{0}=0.15 \mathrm{M} ;[\mathbf{2 a}]$ or $[\mathbf{2 b}]=0.20 \mathrm{M}$; $\left[\mathrm{NaO}-{ }^{t} \mathrm{Bu}\right]=0.225 \mathrm{M} ;\left[\mathrm{Pd}(\mathrm{OAc})_{2}\right]=0.0045 \mathrm{M}$; $[$ binap $]=0.00615$ $\mathrm{M}$; and solvent $=$ toluene. Temperature $=60{ }^{\circ} \mathrm{C}$ (for $2 \mathrm{a}$ ) or $80^{\circ} \mathrm{C}$ (for $\mathbf{2 b}$ ). Isothermal reaction temperatures were chosen based on the previously demonstrated reactivity of the two substrates.

zero-order dependence on both substrate concentrations. Neither reaction shows catalyst deactivation and both exhibit first-order dependence on $\left[\mathrm{Pd}\left(\mathrm{OAc}_{2}\right)\right]$, ${ }^{6}$ allowing us to use the terminology $[\mathrm{Pd}]$ as a surrogate for the total catalyst concentration based on $\left[\mathrm{Pd}\left(\mathrm{OAc}_{2}\right)\right]$. Interestingly, we showed that in competitive reactions, it is the slower hydrazone substrate $\mathbf{2} \mathbf{b}$ that reacts first, being completely consumed prior to the reaction of $2 \mathrm{a}$. These observations led us to propose that the resting state in the reaction employing $2 \mathbf{a}$ is $\mathrm{Pd}$ (binap) and that the reductive elimination complex $\mathbf{6} \mathbf{b}$ is the resting state for the reaction of $\mathbf{2} \mathbf{b}$ (Scheme 2 ). $\mathbf{6} \mathbf{b}$ is more stable than

Scheme 2. Simplified Catalytic Cycle for the Reactions of Scheme 1

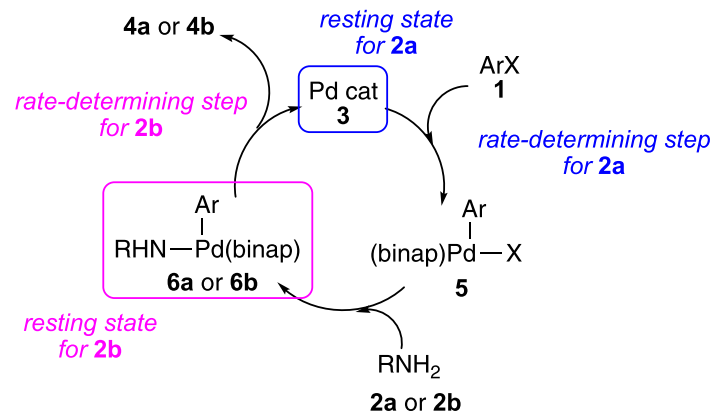

$\operatorname{Pd}$ (binap), enabling competitive reactions of the two amines to be "monopolized" by the pathway through $\mathbf{6 b}$ until substrate $\mathbf{2 b}$ is fully consumed. These earlier mechanistic studies allow us to illustrate the TSR protocol using well-understood model systems with well-defined rate-determining elementary steps. The results of the TSR investigations presented here provide insights into the transition-state requirements for the reaction of each substrate.

The concept of TSRs involves increasing the reaction temperature in a controlled fashion while simultaneously monitoring the reaction progress over the course of a reaction, as shown in Figure 2. The starting temperature for a TSR run is chosen at a value below which significant conversion takes place in order to provide a baseline signal (ca. 35 and $55{ }^{\circ} \mathrm{C}$ for the reactions of $\mathbf{2 a}$ and $\mathbf{2} \mathbf{b}$, respectively), and the temperature ramp proceeds until the full conversion is reached. We employ reaction calorimetry as a kinetic probe, which measures the instantaneous heat flow $\dot{q}$ of the reaction. Heat flow is proportional to the reaction rate $r$ (units of molarity/time) as

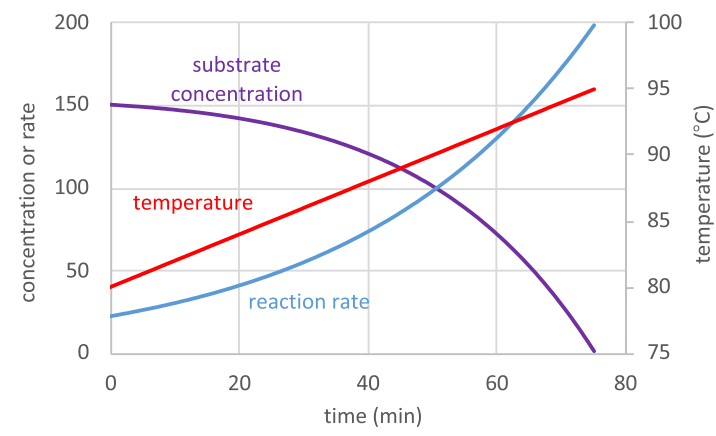

Figure 2. Model plot of a TSR experiment showing the substrate concentration, reaction rate, and temperature as a function of time.

shown in eq 1 , where $\dot{q}$ is the heat flow (units of energy/time), $\Delta H_{\mathrm{rxn}}$ is the thermodynamic heat of reaction (units of energy/ $\mathrm{mol})$, and $V$ is the reaction volume. ${ }^{8}$ Each data point of $(q, t)$ thus represents a separate measurement of the reaction rate at a given substrate concentration and a given temperature.

$$
\dot{q}=\Delta H_{\mathrm{rxn}} \cdot V \cdot r
$$

For multistep reactions, an Arrhenius plot of $\ln (k)$ versus 1/ $T$ provides an activation energy that represents an empirical description of the global multistep reaction. By contrast, an Eyring plot of $\ln (k / T)$ versus $1 / T$ is derived from transitionstate theory and is specifically applicable to the activation parameters associated with the transition barrier to a single elementary step. For catalytic reactions that exhibit a dominant rate-determining step, we can make the assumption that an Eyring plot for such a reaction will provide a description of the activation parameters for this elementary step. ${ }^{9}$ In the case of the reactions of Scheme 1, as shown in the mechanism of Scheme 2, we have previously reported that the oxidative addition of 1 to $\operatorname{Pd}($ binap) 3 is rate-determining when the coupling partner is $\mathbf{2 a}$, while reductive elimination of product $\mathbf{4 b}$ becomes rate-determining when $\mathbf{2 b}$ is the amine employed. ${ }^{6}$ The presence of a definitive elementary step as rate-determining in these two cases allows us to analyze the elementary step activation parameters from our global TSR data. Our work also addresses the question of how the TSR protocol can help provide an insight into cases where the ratedetermining step is not known, is ill-defined, or changes over the range of concentrations and temperatures employed.

\section{RESULTS AND DISCUSSION}

The reactions of Scheme 1 were carried out at three different temperature-ramp rates, otherwise using the conditions shown in Figure 1. ${ }^{7}$ Figure 3 shows the results for the reaction of amines $\mathbf{2 a}$ (top) and $\mathbf{2 b}$ (bottom) with the reactions run at 0.1 , 0.2 , and $0.3{ }^{\circ} \mathrm{C} / \mathrm{min}$. The main plots show reaction rate versus time, and the insets show the same data as a function of temperature.

The elementary step and the respective transition-state complex for the two reactions are given by eq 2 for $\mathbf{2 a}$ and eq 3 for $\mathbf{2 b}$. For the reaction of $\mathbf{2 a}$, the rate at each point is divided by $[1]$ and $[3]$ to obtain the observed rate constant, $k_{2 \mathrm{a}}^{\prime}$. For the reaction of 2 , the rate is divided by [6b] to obtain $k_{2 \mathbf{b}}^{\prime}$. Because each reaction exhibits a definitive resting state, we may assign the concentration of the catalytic Pd intermediate as the total catalyst concentration $\left[\mathrm{Pd}(\mathrm{OAc})_{2}\right]_{\text {total }}$. 

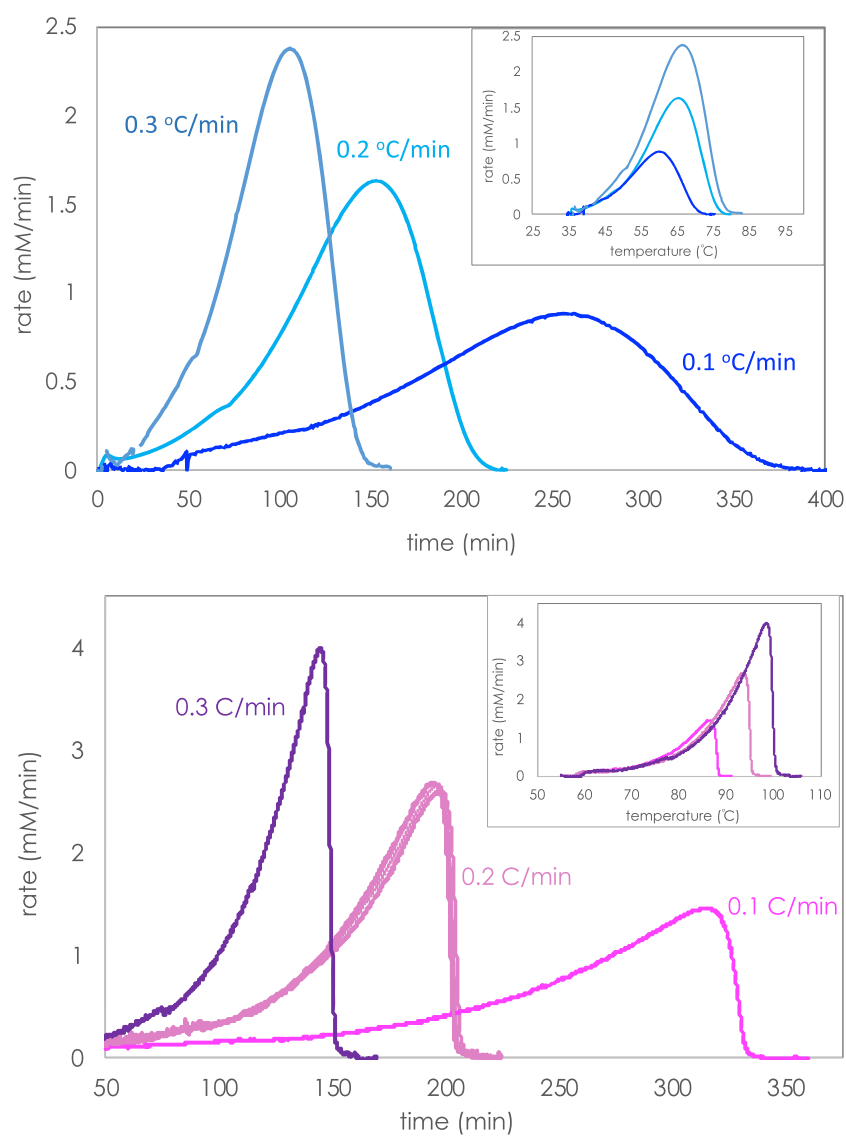

Figure 3. TSR rate profiles vs time for the reaction of Scheme 1 with 2a (top) and $\mathbf{2 b}$ (bottom) under the conditions given in Figure 1, with temperature ramped at the rates shown in the graph. Insets show the plot of rate vs temperature. In the main plot (bottom), three identical runs are shown at $0.2{ }^{\circ} \mathrm{C} / \mathrm{min}$ to demonstrate the reproducibility of the experimental protocol.

Reaction of 2 a

$$
\mathbf{3}+\mathbf{1} \stackrel{K_{2 \mathrm{a}}^{\prime}}{\rightleftarrows} T S_{2 \mathrm{a}}^{\ddagger} \stackrel{k_{2 \mathrm{a}}}{\longrightarrow} \mathbf{5} \quad k_{2 \mathrm{a}}^{\prime}=\frac{\text { rate }}{[\mathbf{3}] \cdot[\mathbf{1}]}
$$

Reaction of $\mathbf{2 b}$

$$
6 \mathrm{a} \stackrel{K_{2 \mathrm{~b}}^{\prime}}{\rightleftarrows} T S_{2 \mathrm{~b}}^{\ddagger} \stackrel{k_{2 \mathrm{~b}}}{\longrightarrow} 4 \mathrm{~b}+3 \quad k_{2 \mathrm{~b}}^{\prime}=\frac{\text { rate }}{[6 \mathrm{~b}]}
$$

We may use these relationships for the observed rate constants to develop Arrhenius and Eyring plots for the TSR progress data in Figure 3. The Arrhenius plots in Figure 4 show excellent linearity and agreement between reactions carried out at different temperature ramp rates, confirming the validity of the TSR experimental protocol. Interestingly, while the reaction rate for $\mathbf{2 a}$ is significantly higher than that for $\mathbf{2 b}$ under identical conditions, the faster reaction exhibits a slightly higher activation energy (135 vs $110 \mathrm{~kJ} / \mathrm{mol}$ ).

While the Arrhenius relationship is empirically derived and is often employed in multistep reactions where a definitive resting state may not be known a priori, or may not exist, the Eyring equation (eq 4) was developed based on the activated complex theory, and it describes the elementary step rate constant in terms of a pre-equilibrium between the reactants and the transition-state complex $K^{\ddagger}$, a transmission coefficient $\kappa$ (typically taken to be equal to 1 ), the Boltzmann constant

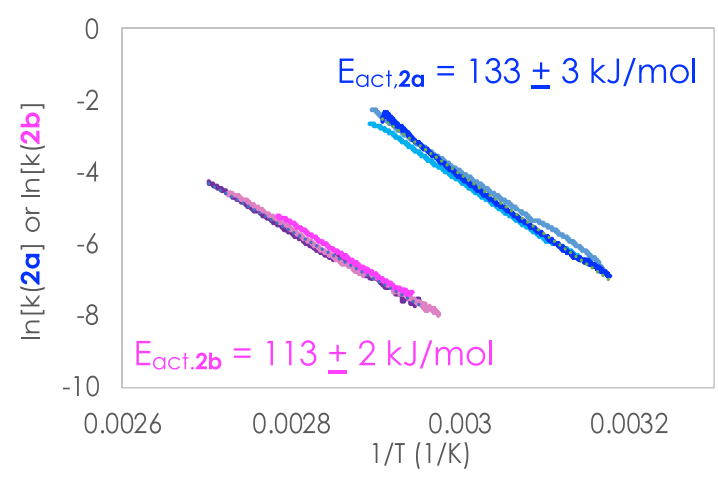

Figure 4. Arrhenius plots constructed from the data in Figure 3 for the reaction of Scheme 1 with $\mathbf{2 a}$ (blue) or $\mathbf{2 b}$ (pink) carried out using the TSR protocol at three different temperature-ramp rates, with the colors for ramp rates as in Figure 3.

$\left(k_{\mathrm{B}}=1.381 \times 10^{-23} \mathrm{~J} \cdot \mathrm{K}^{-1}\right)$, and Planck's constant $(h=6.626 \times$ $\left.10^{-34} \mathrm{~J} \cdot \mathrm{s}\right)$. Defining $K^{\ddagger}$ in terms of activation energy $\Delta G^{\ddagger}=$ $\Delta H^{\ddagger}-T \Delta S^{\ddagger}$, eq 4 is used to construct plots of $\ln (k / T)$ versus $1 / T$ from the TSR data in Figure 3, which are shown in Figure 5. The activation enthalpy and entropy are calculated from the

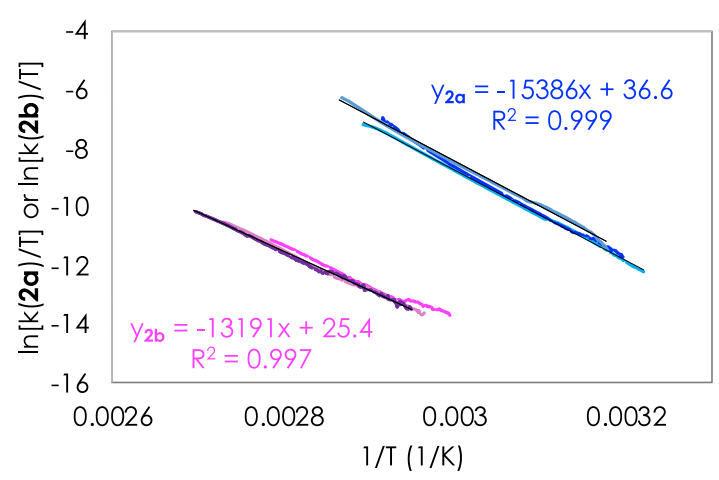

Figure 5. Eyring plots constructed from the data in Figure 3 for the reaction of Scheme 1 with $\mathbf{2 a}$ (blue) or $\mathbf{2 b}$ (pink) carried out using the TSR protocol at three different temperature-ramp rates, with colors for ramp rates as in Figure 3.

slope and intercept of the Eyring plot, as given in eqs 5 and 6, respectively. Table 1 lists these activation parameters averaged from the three runs for each substrate $\mathbf{2 a}$ and $\mathbf{2} \mathbf{b}$.

$$
\begin{gathered}
k=k^{\prime} K^{\ddagger}=\frac{\kappa k_{\mathrm{B}}}{h} \cdot T \cdot \exp \left(\frac{-\Delta G^{\ddagger}}{R T}\right)=\frac{\kappa k_{\mathrm{B}}}{h} \\
\cdot T \cdot \exp \left(\frac{-\Delta H^{\ddagger}+T \Delta S^{\ddagger}}{R T}\right)
\end{gathered}
$$

Reaction enthalpy

$$
\Delta H^{*}=(\text {-slope }) \cdot R
$$

Reaction entropy

Table 1. Activation Parameters Derived from the TSRs of Figure 3

$\begin{array}{ccr}\text { amine } & \Delta H^{\ddagger}(\mathrm{kJ} / \mathrm{mol}) & \left.\Delta S^{\ddagger} \text { (e.u. }\right) \\ \text { 2a } & 131 \pm 3 & 29.4 \pm 2.5 \\ \text { 2b } & 110 \pm 2 & 3.5 \pm 1.2\end{array}$




$$
\Delta S^{\ddagger}=R \cdot(y \text { intercept }-23.76)
$$

These calculations show that the activation enthalpy is similar for the two reactions, as was also observed for the global activation energy. In addition, these activation parameters closely match the values reported for $\mathbf{2 b}$ in the Buchwald-Hartwig amination reaction using Pd catalysts with other biaryl phosphine ligands. ${ }^{10}$ The key difference between the reactions of the two amines lies in the activation entropy. While the activation entropy change is near zero for substrate $\mathbf{2 b}$, the reaction with substrate $\mathbf{2 a}$ exhibits an order of magnitude larger change in activation entropy. The large positive entropy change for $\mathbf{2 a}$ accounts for its faster rate as it lowers $\Delta G^{\ddagger}$ much more than in the case of $\mathbf{2 b}$. Figure 6 shows

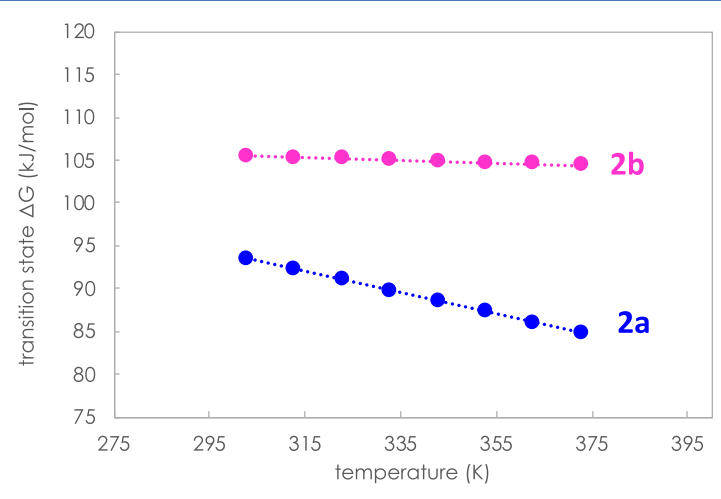

Figure 6. Activation free energy as a function of temperature for the reactions of Scheme 1, as calculated from the activation parameters given in Table 1.

the relationship between $\Delta G^{\ddagger}$ and the reaction temperature for the reactions of $\mathbf{2 a}$ and $\mathbf{2 b}$, as calculated from the activation parameters. This difference in $\Delta G^{\ddagger}$ and, therefore, in the rate between the two reactions is enhanced as the temperature is increased. The greater role that entropy changes play in the reaction of $2 \mathrm{a}$ also helps to rationalize the observation of higher activation energy, as is shown in Figure 4, because $E_{\text {act }}$ is an enthalpic term. It is important to note that accurate measurement of activation entropy relies on accurate knowledge of the concentration of the active catalyst species in the elementary step in question, either $\mathbf{3}$ or $\mathbf{6} \mathbf{b}$ (in the case of the reaction of $\mathbf{2 a}$ or $\mathbf{2 b}$, respectively), as well as on accurate and reproducible kinetic data because extrapolation to the $y$ intercept can result in different values for relatively small differences in the slope. ${ }^{11}$

At first glance, it may seem curious that the unimolecular reaction of $\mathbf{6 b}$ exhibits a lower activation entropy than the bimolecular reaction of $\mathbf{3}+\mathbf{1} \rightarrow \mathbf{5}$, as it was conventionally proposed that the action of bringing two reactants together should reduce the entropy, compared with the unimolecular conversion of one species to another. Solvent effects may help to rationalize this result. The solvent choice in $\mathrm{Pd}$-catalyzed cross-coupling reactions and in metal-complex formation has been studied and reviewed extensively. ${ }^{9-22}$ Similar findings were reported for the oxidative addition of aryl halides to lowligated $\operatorname{Pd}(0)$ complexes by Amatore and coworkers, where an unusually high activation entropy of 24.1 e.u. was attributed to the strong coordination of halide ions to a Pd complex derived from $\mathrm{Pd}(\mathrm{PPh} 3)_{4}{ }^{18}$ Strongly polarizable solvents such as toluene can stabilize molecules or ions containing induced or permanent dipoles. A recent computational study showed that while $\mathrm{Pd}(0)$ (binap) is a coordinatively unsaturated 14-electron complex that is likely present as a solvated species, $\mathrm{Pd}(\mathrm{II})$ (binap) $\operatorname{ArX}$ is a 16-electron complex with four occupied coordination sites (bidentate ligand, Ar, and X), making it less accessible to solvent. ${ }^{16}$ Thus, the coordination and subsequent oxidative addition of $\operatorname{ArBr} 1$ to 3 would be accompanied by the release of solvent molecules from the catalyst, giving rise to a greater entropic gain in the reaction of $\mathbf{2 a}$ compared to $\mathbf{2 b}$. The unimolecular elementary rate-determining step for $\mathbf{2 b}$ should be less sensitive to solvent interactions, resulting in a smaller change in entropy for that reaction. Indeed, as mentioned above, the activation parameters determined for the reaction of $\mathbf{2 b}$ are in agreement with those recently reported for Buchwald-Hartwig reactions in which reductive elimination was found to be the rate-determining step. ${ }^{10}$

A comparison of the TSR profiles for the reactions of the two amines may provide suggestions for process improvements. The change in the resting state in the case of the reaction of $\mathbf{2 b}$ that causes the stabilization of intermediate $\mathbf{6 b}$ leads to an overall slower reaction compared to that of $2 a$, but this system may be more robust than the reaction of $2 a$, where the resting state is a 14-electron complex $\mathrm{Pd}$ (binap). Solvation of this complex helps to stabilize it, but the system may be intrinsically more predisposed to catalyst deactivation in the elementary reaction of eq 2 than that of eq 3, where sites on the catalyst are not much exposed to solvent. This analysis of activation parameters may also help rationalize the solvent effects in these coupling reactions. Shifting of the catalyst resting state and stabilization of a late-occurring intermediate species by interrogating solvent effects may become a useful strategy in rational approaches to increase the catalyst robustness.

Each of the two reactions under study maintain the resting state and the rate-determining step defined by eqs 2 and 3 over the entire reaction progress. In many catalytic reactions, however, shifting of the catalyst resting state concomitant with a shift in the rate-determining step occurs over the course of the reaction, which is a feature of many isothermal catalytic reactions exhibiting saturation kinetics. This is why it is generally recommended that determination of activation parameters (typically using isothermal initial rate measurements) be made using the same reactant concentration at each temperature. The temperature-scanning protocol follows the entire reaction progress, which means that both temperature and concentrations are changing. The success of the TSR protocol relies on deconvolution of the dependence of the reaction rate on reactant concentrations by normalizing the rate by the reactant driving forces. In the case of the reactions of $\mathbf{2} \mathbf{a}$ and $\mathbf{2 b}$, this normalization was straightforward according to eqs 2 and 3. The catalytic rate law can, however, be more complex in the case of saturation kinetics, and RPKA handles this by approximating the catalytic rate expression with a power law form that typically exhibits noninteger orders in substrate concentration. For example, given a simple Michaelis-Menten rate expression for the reaction of substrate $\mathbf{A}$ in eq 7a, Figure 7 illustrates how a power law form of the rate expression as in eq $7 \mathrm{~b}$ can approximate the true steadystate catalytic rate law over a defined concentration range. ${ }^{23}$ The form of the two profiles coincides with the values of $m$ between 0 and 1 approximating the values of $K_{\text {eq }}$, with increasing $K_{\text {eq }}$ corresponding to smaller values of $m$. 


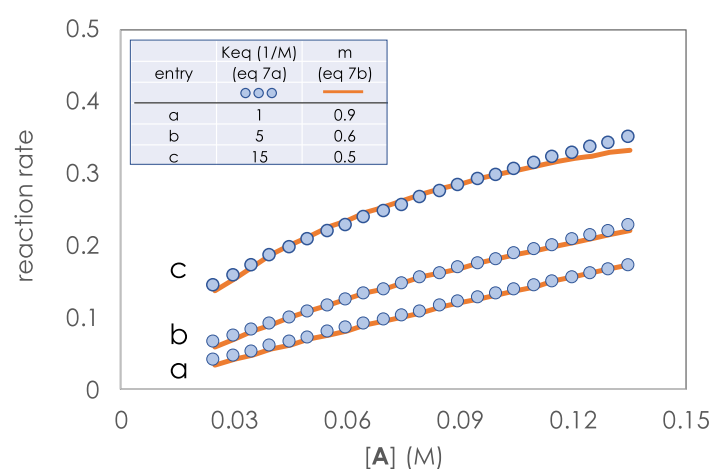

Figure 7. Illustration of how the power law with an order $m$ (eq $7 \mathrm{~b}$ ) approximates the Michaelis-Menten reaction rate expression (eq 7a) with the equilibrium constant $K_{\text {eq }}$ over a defined range of [A].

$$
\begin{aligned}
& \text { Rate }=k_{\mathrm{MM}} \cdot \frac{[\mathbf{A}]}{1+K_{\mathrm{eq}}[\mathbf{A}]} \\
& \text { Rate }=k_{\text {power }} \cdot[\mathbf{A}]^{m}
\end{aligned}
$$

In a case where neither $m$ nor $K_{\text {eq }}$ is known a priori, the temperature-scanning protocol may also be used to determine the concentration dependencies of substrates and catalysts, which are typically explored in isothermal experiments, including the classical initial rate experiments, RPKA protocols of the same [excess] and different [excess] experiments, ${ }^{1}$ and variable time normalization analysis. ${ }^{24}$ The key is to exploit an overlay in TSR experiments that plot empirical $k_{\text {obs }}$ values after proposing values for reaction orders. For example, in a reaction following the Michaelis-Menten rate law of eq 7a, the appropriate integer or noninteger value of $\mathbf{m}$ will be the value that provides an overlay in Arrhenius plots of $\ln \left[k_{\mathrm{obs}}(T)\right]$, as given in eq $8 b$ constructed from the reactions carried out at different temperature-ramp rates.

$$
\begin{aligned}
& \text { Rate }=k_{\text {obs }}(T) \cdot[\mathbf{A}]^{m} \\
& \ln \left[k_{\text {obs }}(T)\right]=\ln \left[\frac{\text { rate }}{[\mathbf{A}]^{m}}\right]
\end{aligned}
$$

As an example, we can consider the bimolecular elementary reaction that is rate-determining in the case of reaction of $2 a$, as given in eq 2. The Arrhenius plots of Figure 4 plotting $\ln \left(k_{\text {obs }}\right)$ versus $1 / T$ show an overlay for three reactions carried out at three different temperature-ramp rates, where $k_{\text {obs }}$ equals the rate normalized by the substrate concentrations, with $m=$ 1. Figure 8 reveals the Arrhenius plots that would result by simply plotting the rate without normalizing for the concentration dependence of $\mathbf{1}$. The three TSR profiles deviate from linearity and do not display an overlay at higher temperatures, indicating that the true substrate concentration dependencies have not been properly deconvoluted from the temperature dependence. Iteration of eq $8 b$ using different values of $m$ may be carried out until Arrhenius plots of reactions carried out at different temperature ramp rates become linear and overlay over the broad temperature range of the reaction.

Failure to find a value of $m$ that gives linear Arrhenius plots and an overlay between runs at different ramp rates may indicate that the concentration range of the reaction is too broad to be described by a single power law form or that a single set of activation parameters cannot describe the entire

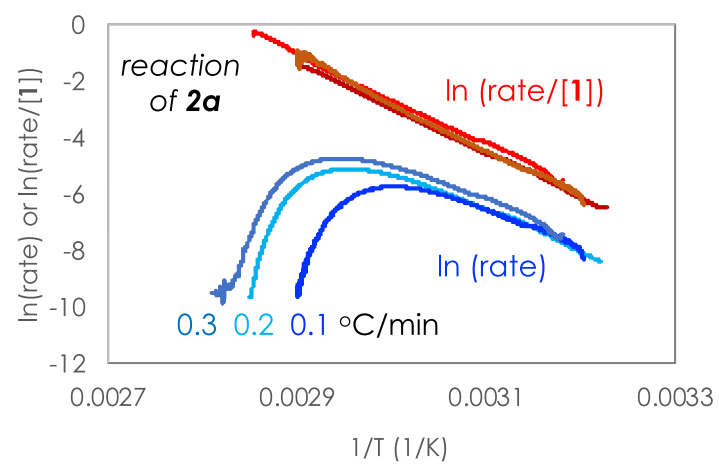

Figure 8. Arrhenius plots of three TSR experiments for the reaction of $\mathbf{2 a}$ at three different ramp rates from Figure 3 with the data plotted as $y=\ln$ (rate) (in blue), without first normalizing for the rate dependence of $[\mathbf{1}]$. The rate normalized by $[\mathbf{1}]$ for the same three reactions is shown in red for comparison. The rate has not been normalized by the catalyst concentration, which is the same for all runs and is lumped into $k_{\text {obs }}$ in eqs $8 \mathrm{a}$ and $8 \mathrm{~b}$.

concentration and temperature range under study. Preparing several plots from reaction data extracted at different defined concentration ranges can help to define the point at which the rate-determining step shifts as a function of both temperature and concentration. Obtaining such information from isothermal reactions would require a significantly larger number of isothermal initial rate experiments, with multiple reactions carried out at different initial substrate concentrations and different reaction temperatures. The treatment in Figure 8 and eq $8 \mathrm{~b}$ demonstrates that the TSR protocol allows the determination of both substrate concentration dependencies and activation parameters from a minimal number of separate experiments.

\section{CONCLUSIONS}

A recently developed temperature-scanning protocol is applied to the Pd-catalyzed Buchwald-Hartwig $\mathrm{C}-\mathrm{N}$ coupling reaction using two different amine substrates. The concentration and temperature data are collected for the course of the reaction that is carried out using a linear temperature-ramp rate. The global activation energy may be determined by constructing Arrhenius plots of $\log \left(k_{\text {obs }}\right)$ versus $1 / T$. The rate at any given point is normalized by substrate concentration dependencies. The correct order in the substrate concentration is revealed when an overlay is observed between profiles of reactions at different temperature-ramp rates. This protocol allows both reaction rate laws and temperature-dependent activation parameters to be calculated from a minimal number of experiments.

For cases where one elementary step in a multistep catalytic network is revealed to be rate-determining, TSR profiles may also be used to construct Eyring plots to determine the activation parameters $\Delta G^{\ddagger}, \Delta H^{\ddagger}$, and $\Delta S^{\ddagger}$. Treatment of the TSR data from the reaction of the amine substrates $\mathbf{2 a}$ and $\mathbf{2 b}$ revealed that while the reaction of $\mathbf{2 a}$ is significantly faster, it also exhibits a much stronger dependence on the change in the activation entropy than does the reaction of $\mathbf{2} \mathbf{b}$, which is dominated by enthalpic factors. The larger magnitude of $\Delta S^{\ddagger}$ for the reaction of $\mathbf{2} \mathbf{a}$ is attributed to the need to desolvate the $\mathrm{Pd}$ (binap) catalyst prior to oxidative addition of the aryl halide substrate 1. This TSR protocol may aid in the catalyst and reaction design by suggesting particular catalyst-resting states that improve the catalyst robustness. 


\section{ASSOCIATED CONTENT}

\section{(s) Supporting Information}

The Supporting Information is available free of charge at https://pubs.acs.org/doi/10.1021/acscatal.0c01929.

Details about the experimental reaction, analytical procedures, and data manipulations (PDF)

\section{AUTHOR INFORMATION}

\section{Corresponding Author}

Donna G. Blackmond - Department of Chemistry, Scripps Research, La Jolla, California 92037, United States; 다이.org/0000-0001-9829-8375; Email: blackmond@ scripps.edu

\section{Author \\ Olivia P. Schmidt - Department of Chemistry, Scripps Research, La Jolla, California 92037, United States}

Complete contact information is available at:

https://pubs.acs.org/10.1021/acscatal.0c01929

\section{Notes}

The authors declare no competing financial interest.

\section{ACKNOWLEDGMENTS}

D.G.B. acknowledges support from the Scripps Research and the US National Science Foundation (CHE-192443). O.P.S. acknowledges funding from the Swiss National Science Foundation.

\section{REFERENCES}

(1) (a) Blackmond, D. G. Reaction Progress Kinetic Analysis: A Powerful Methodology for Mechanistic Studies of Complex Catalytic Reactions. Angew. Chem. Int. Ed. 2005, 44, 4032-4320. (b) Mathew, J. S.; Klussmann, M.; Iwamura, H.; Valera, F.; Futran, A.; Emanuelsson, E. A. C.; Blackmond, D. G. Investigations of PdCatalyzed ArX Coupling Reactions Informed by Reaction Progress Kinetic Analysis. J. Org. Chem. 2006, 71, 4711-4722. (c) Blackmond, D. G. Kinetic Profiling of Organic Catalytic Reactions as a Mechanistic Tool. J. Am. Chem. Soc. 2015, 137, 10852-10866.

(2) Schmidt, O. P.; Dechert...Schmitt, A. M.; Garnsey, M. R.; Wisniewska, H. M.; Blackmond, D. G. Kinetic Analysis of Catalytic Organic Reactions Using a Temperature Scanning Protocol. ChemCatChem 2019, 11, 3808-3813.

(3) (a) Wojciechowski, B. W. The Temperature Scanning Reactor: A Kinetics Instrument. Chem. Eng. Commun. 2003, 190, 1115-1131. (b) Landau, R. N.; Blackmond, D. G.; Tung, H.-H. Calorimetric Investigation of an Exothermic Reaction: Kinetic and Heat Flow Modeling. Ind. Eng. Chem. Res. 1994, 33, 814-820.

(4) (a) Aroh, K. C.; Jensen, K. F. Efficient Kinetic Experiments in Continuous Flow Microreactors. React. Chem. Eng. 2018, 3, 94-101. (b) Waldron, C.; Pankajakshan, A.; Quaglio, M.; Cao, E.; Galvanin, F.; Gavriilidis, A. An Autonomous Microreactor Platform for the Rapid Identification of Kinetic Models. React. Chem. Eng. 2019, 4, $1623-1636$.

(5) (a) Buchwald, S. L.; Mauger, C.; Mignani, G.; Scholz, U. Industrial...Scale Palladium...Catalyzed Coupling of Aryl Halides and Amines -A Personal Account. Adv. Synth. Catal. 2006, 348, 23-39. (b) Hartwig, J. F. Handbook of Organopalladium Chemistry for Organic Synthesis; Negishi, E. I., Ed.; Wiley-Interscience: New York, 2002; Vol. 1, p 1051-1096; (c) Muci, A. R.; Buchwald, S. L. Practical Palladium Catalysts for C-N and C-O Bond Formation. Top. Curr. Chem. 2002, 219, 131-209. (d) Alcazar-Roman, L. M.; Hartwig, J. F.; Rheingold, A. L.; Liable-Sands, L. M.; Guzei, I. A. Mechanistic Studies of the Palladium-Catalyzed Amination of Aryl Halides and the Oxidative Addition of Aryl Bromides to Pd(BINAP)2 and Pd(DPPF)2: An
Unusual Case of Zero-Order Kinetic Behavior and Product Inhibition. J. Am. Chem. Soc. 2000, 122, 4618-4630. (e) Singh, U. K.; Strieter, E. R.; Blackmond, D. G.; Buchwald, S. L. Mechanistic Insights into the Pd(BINAP)-Catalyzed Amination of Aryl Bromides: Kinetic Studies under Synthetically Relevant Conditions. J. Am. Chem. Soc. 2002, 124, 14104-14114. (f) Shekhar, S.; Ryberg, P.; Hartwig, J. F.; Mathew, J. S.; Blackmond, D. G.; Strieter, E. R.; Buchwald, S. L. Reevaluation of the Mechanism of the Amination of Aryl Halides Catalyzed by BINAP-Ligated Palladium Complexes. J. Am. Chem. Soc. 2006, 128, 3584-3591.

(6) Ferretti, A. C.; Mathew, J. S.; Ashworth, I.; Purdy, M.; Brennan, C.; Blackmond, D. G. Mechanistic Inferences Derived from Competitive Catalytic Reactions: $\mathrm{Pd}$ (binap)-Catalyzed Amination of Aryl Halides. Adv. Synth. Catal. 2008, 350, 1007-1012.

(7) See Supporting Information for details.

(8) LeBlond, C.; Wang, J.; Larsen, R. D.; Orella, C. J.; Forman, A. L.; Landau, R. N.; Laquidara, J.; Sowa, J. R.; Blackmond, D. G.; Sun, Y.K. Reaction Calorimetry as a Powerful Tool for Characterizing Complex Reactions. Thermochim. Acta 1996, 289, 189-207.

(9) Brezonik, P. L. Chemical Kinetics and Process Dynamics in Aquatic Systems; Lewis Publishers, CRC Press: Boca Raton, 2002, Chapter 3, pp 144-145.

(10) Arrechea, P. L.; Buchwald, S. L. Biaryl Phosphine Based Pd(II) Amido Complexes: The Effect of Ligand Structure on Reductive Elimination. J. Am. Chem. Soc. 2016, 138, 12486-12493.

(11) Lente, G.; Fábián, I.; Poë, A. J. A Common Misconception About the Eyring Equation. New J. Chem. 2005, 29, 759-760.

(12) Wolfe, J. P.; Buchwald, S. L. Scope and Limitations of the Pd/ BINAP-Catalyzed Amination of Aryl Bromides. J. Org. Chem. 2000, 65, 1144-1157.

(13) Lyngvi, E.; Sanhueza, I. A.; Schoenebeck, F. Dispersion Makes the Difference: Bisligated Transition States Found for the Oxidative Addition of $\mathrm{Pd}\left(\mathrm{P}_{t} \mathrm{Bu}_{3}\right)_{2}$ to $\mathrm{Ar}-\mathrm{OSO}_{2} \mathrm{R}$ and Dispersion Controlled Chemoselectivity in Reactions with $\mathrm{Pd}\left[\mathrm{P}(\mathrm{iPr})\left(t \mathrm{Bu}_{2}\right)\right]_{2}$. Organometallics 2015, 34, 805.

(14) Sherwood, J.; Clark, J. H.; Fairlamb, I. J. S.; Slattery, J. M. Solvent Effects in Palladium Catalyzed Cross-Coupling Reactions. Green Chem. 2019, 21, 2164-2213.

(15) Christensen, H.; Kiil, S.; Dam-Johansen, K.; Nielsen, O.; Sommer, M. B. Effect of Solvents on the Product Distribution and Reaction Rate of a Buchwald-Hartwig Amination Reaction. Org. Process Res. Dev. 2006, 10, 762-769.

(16) Anand, M.; Nørskov, J. K. Scaling Relations in Homogeneous Catalysis: Analyzing the Buchwald-Hartwig Amination Reaction. ACS Catal. 2020, 10, 336-345.

(17) Ohrai, K.; Kondo, K.; Sodeoka, M.; Shibasaki, M. Effects of Solvents and Additives in the Asymmetric Heck Reaction of Alkenyl Inflates: Catalytic Asymmetric Synthesis of Decalin Derivatives and Determination of the Absolute Stereochemistry of $(+)$-Vemolepin. $J$. Am. Chem. Soc. 1994, 116, 11737-11748.

(18) Amatore, C.; Azzabi, M.; Jutand, A.; M’Barki, M. A.; Mottier, L. Role and Effects of Halide Ions on the Rates and Mechanisms of Oxidative Addition of Iodobenzene to Low-Ligated Zerovalent Palladium fComplexes $\mathrm{Pd}^{\circ}\left(\mathrm{PPh}_{3}\right)_{2}$. J. Am. Chem. Soc. 1991, 113, 8375-8384.

(19) Nancollas, G. H. The Thermodynamics of Metal-Complex and Ion-Pair Formation. Coord. Chem. Rev. 1970, 5, 379-415.

(20) Ameen, H. M.; Kunsagi-Mate, S.; Bognar, B.; Szente, L.; Poor, M.; Lemli, B. Thermodynamic Characterization of the Interaction between the Antimicrobial Drug Sulfamethazine and Two Selected Cyclodextrins. Molecules 2019, 24, 4565.

(21) Schwarzenbach, G. Electrostatic and Non-Electrostatic Contributions to Ion Association in Solution. Pure Appl. Chem. 1970, 24, 307-334.

(22) Levy, C. J.; Puddephatt, R. J. Rapid Reversible Oxidative Addition of Group 14-Halide Bonds to Platinum(II): Rates, Equilibria, and Bond Energies. J. Am. Chem. Soc. 1997, 119, 10127-10136. 
(23) The rate dependence on [1] is deconvoluted from the rate dependence on temperature by normalizing rate by the instantaneous concentration of $\mathbf{1}$ at each point in time (and temperature). Without correct normalization of the reactant concentration dependence, the Arrhenius plot obtained will not be linear. See Figure 8 and the Supporting Information for details.

(24) Burés, J. Variable Time Normalization Analysis: General Graphical Elucidation of Reaction Orders from Concentration Profiles. Angew. Chem., Int. Ed. 2016, 55, 16084-16087. 